\title{
Characteristics of retinal detachment associated with atopic dermatitis
}

\author{
Youlim Lee \\ Severance Hospital \\ Woo-kyung Park \\ Seoul St. Mary's Hospital \\ Rae-Young Kim \\ Seoul St. Mary's Hospital \\ Mirinae Kim \\ Seoul St. Mary's Hospital \\ Young-Gun Park \\ Seoul St. Mary's Hospital \\ Young-Hoon Park ( $\nabla$ parkyh@catholic.ac.kr) \\ Seoul St. Mary's Hospital
}

\section{Research Article}

Keywords: Atopic dermatitis, retinal detachment, cataract surgery

Posted Date: December 3rd, 2020

DOl: https://doi.org/10.21203/rs.3.rs-107929/v1

License: (c) (i) This work is licensed under a Creative Commons Attribution 4.0 International License.

Read Full License 


\section{Abstract}

Purpose: To examine the characteristics of rhegmatous retinal detachment(RRD) associated with atopic dermatitis.

Study design: retrospective case-control study

\section{Method}

The study was conducted between 2008 and 2018, whereby 2257 patients underwent RD surgery at this clinic. Among them, 61 patients who have been diagnosed as atopy were treated as experimental group, and 100 patients who didn't have atopy were randomly selected as control group.

MAIN OUTCOME MEASURES: In this study, demographics, characteristics of detachment, initial operative method, and prognosis after surgery were investigated. Additionally, in pseudophakic RD patients, the period between the cataract surgery and onset of RD was measured.

Result: In atopy group, postoperative VA and prognosis were significantly worse and bilateral involvement of RD was more common than control group. However, it is notable that there was no difference of mean preoperative VA and refractive index between two groups. There was a difference in characteristics of retinal breaks in two groups. Additionally, it was noted that in pseudophakic patients, the risk of developing RD within 1 year after cataract surgery was significantly higher in atopic group than in control group.

Conclusion: Generally speaking, RD in atopy occurs at a young age and has different characteristics noted than with non-atopic patients. In this context, atopic RD has poor visual prognosis, and for this reason it requires careful management. Furthermore, the risk of developing RD within 1 year after cataract surgery is higher in atopy, and thus we suggest regular and extensive check-up after cataract surgery especially in atopic patients.

\section{Introduction}

To begin with, atopic dermatitis is a common disease with a worldwide prevalence of $15-25 \%$ in children and $1-3 \%$ in adults, and it has been reported to have a similar prevalence in Korea.[1]

According to previous study, atopic patients have 2 to 12 times more ocular disease such as dry eye, keratoconjunctivitis, keratoconus, retinal detachment, glaucoma, and cataract compared with the incidence in a non-atopic population. In particular, retinal detachment occurs 3.22 times more than in the general population.[2]

Generally speaking, RD is a complication that can cause severe visual loss and it occurs at a frequency of 8-10\% in patients with atopic dermatitis between the ages of 10 and 30.[3-5] It is known to develop at a younger age and have poor prognosis as compared with a population without atopic dermatitis.[6, 7] 
The causes of atopy-related RD have not been elucidated yet, but there are many theories; RD might be caused by atopic edema induced by allergies or blood vessel abnormalities which are associated with atopic dermatitis,[8] and diseased vitreous can cause retinal break due to immune response,[9] or the most widely accepted theory is that the frequent rubbing of the eye in patients with atopy might lead to traction and retinal tear of the anterior vitreous, resulting in RD. There is also a study that the incidence of $\mathrm{RD}$ is about 8 times higher in people with frequent eye rubbing.[10]

It is noted that RD associated with atopy is more frequent in bilateral involvement than associated with congenital, inflammatory, or high myopia, and also related with a higher risk of surgical failure due to PVR.[11]

Although the risk of RD in atopy patients is known to be high, there have been few studies on the clinical characteristics of atopy RD and association with cataract surgery is unknown. Therefore, we are going to investigate the frequency and clinical characteristics of atopic RD in patients who have been followed up for a relatively long time in a single institution

The purpose of our study was to identify the characteristics of RD associated with atopic dermatitis during 10 years, and to compare with non-atopic RD.

\section{Methods}

The study was conducted between 2008 and 2018, whereby 2257 patients underwent RD surgery in Seoul St. Mary's Hospital, Seoul, Republic of Korea. The medical records of all patients were retrospectively reviewed. Notably, 61 patients with atopy were treated as the experimental group and 100 patients without atopy were treated as the control group. Patients with atopy were previously diagnosed as atopy by a dermatologist. Of the 2197 patients except the atopy RD group, who didn't have atopy, 100 patients were randomly selected as representative of the control group. Of the total RD patients, $2.7 \%$ had atopic dermatitis. Finally, patients with RD due to trauma, exudate, and macular hole was excluded from the study.

\section{Ethical approval and consent to participate}

This study was performed according to the tenets of the Declaration of Helsinki, and the study protocol was approved by the institutional review/ethics boards of the Catholic University of Korea and our hospital. Informed consent was waived owing to the retrospective nature of this study, and patient records were anonymized prior to all records analysis. All protocols were approved by the Institutional Review and Ethics Boards of Seoul St. Mary's Hospital, South Korea.

All patients in the study underwent a complete preoperative evaluation, which included a comprehensive history taking, best-corrected visual acuity (BCVA) measurement, slit-lamp microbioscopy, fundus examination with a contact wide angle viewing lens (Superquad 160, Volk) that can evaluate range, type and location of retinal break, optical coherence tomography to confirm macular involvement, presence of 
proliferative vitreoretinopathy (PVR) (those classified as Grade 3 or above according to the classic classification of the Retina Society)[12].

We classified break type into 6 categories; retinal dialysis, horseshoe tear, retinal tear (as tear except horseshoe tear), hole with lattice, hole without lattice, and if the type of break is unclear, it was classified as an unknown type. Additionally, we also classified the location of the break into 5 categories; superotemporal, inferotemporal, superonasal, inferonasal, or unknown.

=Generally speaking, the BCVA and refraction were compared before surgery, and refraction was excluded if they were in a pseudophakic state. In eyes with a history of cataract surgery, the mean period between the cataract surgery and diagnosis of RD was recorded.

The range of the area in which RD involved was counted by the number of quadrants.

In all patients, the surgeon decided the surgical procedure by considering fundus and lens status, age, location of tear, PVR. Here, the surgery was performed either by the procedure of silicone sponge buckling or encircling, 23 or 25 gauge pars plana vitrectomy, or both. Likewise, if it was necessary, additional subretinal drainage, air-gas exchange, silicone oil implantation, and laser photocoagulation were performed if required in this case.

To evaluate an anatomical and functional outcome, the postoperative BCVA and recurrence rate were investigated at 6 months and last FU after surgery.

In subjects with RD both eyes, each eye was recorded separately. But in two cases of the atopy group, the opposite eye was a localized RD and cured only with the use of a barrier laser, and for that reason those two subjects were excluded. Among the evaluated bilateral patients, if the operation of the other eye performed in other hospital, the other eye was excluded in the study; one case in each group.

$\underline{\text { Statistical analysis }}$

To begin with, for the VA analysis, we converted the logarithm to the minimum Angle of Resolution (log MAR) VA for statistical analysis. The VA of counting fingers, hand movements, light perception were then assigned ratios of $0.01,0.001,0.0005$, respectively. In addition, a Chi-square test, Mann-Whitney test, and paired t-test were used as statistical testing tools in this study. Therefore, all statistical analyses were performed using SPSS statistical software for Windows, version 25.0 (SPSS, Chicago, IL) The statistical significance level was set at $P<0.05$.

\section{Results}

In this study, there were a total of 2257 patients who had RD surgery in our clinic between 2008 and 2018 . Of these, 61 patients had atopic dermatitis and it accounts for $2.7 \%$ of total RRD patients.

It is noted that the demographics of the study participants were presented in Table 1. 
Table 1. Demographic and clinical characteristics of the study participants.

\begin{tabular}{lccc}
\hline & Atopy & Non-atopy & P value \\
\hline $\begin{array}{l}\text { Demographics } \\
\text { No. of patients (eyes) }\end{array}$ & $61(71)$ & $100(100)$ & \\
\hline Sex - male:female (\%) & $39: 22(64: 36)$ & $63: 37$ & 0.811 \\
\hline Mean age (year) & $23.08 \pm 10.24$ & $52.29 \pm 15.48$ & $\mathbf{0 . 0 0 0 *}$ \\
\hline Involved eye - OD:OS (\%) & $37: 34(52: 48)$ & $50: 50$ & 0.785 \\
\hline Preop VA (Log MAR) & 1.14 & 0.96 & 0.294 \\
\hline Number of bilateral involved patients: $\mathrm{n}(\%)$ & $13(21 \%)$ & $1(1 \%)$ & $\mathbf{0 . 0 0 0 *}$ \\
\hline $\begin{array}{l}\text { Lens status; } \\
\text { phakic: pseudophakic (\%) }\end{array}$ & $43: 28(60: 40)$ & $74: 26$ & 0.063 \\
\hline
\end{tabular}

Values are presented as mean \pm SD unless otherwise indicated.

$\mathrm{VA}=$ visual acuity

*p-value by Mann-Whitney U test and chi-square test

Of the total 61 patients with atopy, 13 patients had bilateral RD. Two of them were seen to have localized $\mathrm{RD}$ and treated only with barrier laser, and for that reason they were not included in the study. Additionally, 1 patient received RRD surgery from another hospital, and for that reason the opposite eye was excluded from the experimental group. Therefore, it is noted that a total of 71 eyes were included in the atopy group.

As was seen, of the 100 control patients, only one patient had bilateral RD. However, the other eye was received RD surgery from another hospital, and therefore not included in the study. In this respect, a total of 100 eyes were included in the control group.

Thus, the rate of bilateral RD was $21 \%$ in the atopic group and $1 \%$ in the control group. Notably speaking, the rate of bilateral involvement was significantly higher in the atopic group (P value 0.000$)$.

In this study, there were 39 males (64\%) and 22 females (36\%) in the atopy group, and 63 males (63\%) and 37 females (37\%) in non-atopy group ( $P$ value 0.811 ).

In this respect, the average age was $23.08 \pm 10.24$ years in the atopy group, and $52.29 \pm 15.48$ years in nonatopy group ( $P$ value 0.000 ). Additionally, the mean age was statistically significantly younger in atopy group (Table 1, Figure 1).

In the atopy group, 37 had RD in the right eye and 34 had RD in the left eye. In the non-atopy group, 50 had $\mathrm{RD}$ in right eye and 50 in left eye. Likewise, in both groups, a similar rate occurred in both eyes. There 
was also no statistically significant difference in the lens status (phakic or pseudophakic) between the two groups.

In this case, the mean spherical equivalent before surgery was $-3.09 \pm 0.392$ in the atopic group and -3.56 \pm 0.372 in the control group, there was no statistical difference (P value 0.953, Table 2).

\section{Table 2. Refractive status before surgery.}

\begin{tabular}{llll}
\hline Refractive status & Atopy & Non-atopy & P value \\
\hline Spherical equivalent & $-3.09 \pm 0.392$ & $-3.56 \pm 0.372$ & \\
(Mean \pm SD) & & & \\
hyperopia & $1(1 \%)$ & $7(7 \%)$ \\
emme to -3D & $31(43.7 \%)$ & $44(44 \%)$ \\
Myopia (3D to-8D) & $25(35.2 \%)$ & $3030 \%)$ \\
High myopia(over -8D) & $5(7 \%)$ & $19(19 \%)$ \\
\end{tabular}

Values are presented as mean \pm SD unless otherwise indicated.

Values are presented as $\mathrm{n}(\%)$.

- p-value by Mann-Whitney U test

Here, we also compared initial operative method (Table 3). Next, PPV alone was performed in $21 \%$ (15 eyes) of the atopy group and 59\% (59 eyes) of the control group. Likewise, buckling alone was performed in $17 \%$ (12 eyes) of the atopy and 31\% (31 eyes) of the control. Encircling alone was performed $24 \%$ (17 eyes) in the atopy and $7 \%$ ( 7 eyes) in the control. And in 38\% (27 eyes) of the atopy and $3 \%$ of the control, encircling and $\mathrm{ppV}$ were performed at the same time as an initial surgery. Therefore, in the atopic group, two procedures were performed together as an initial surgery, which was much more than the control group.

Table 3. Initial method of surgery in each group. 


\begin{tabular}{lcc}
\hline & Atopy & Non-atopy \\
\hline Initial OP method & $15(21 \%)$ & $59(59 \%)$ \\
Pars plana vitrectomy & $12(17 \%)$ & $31(31 \%)$ \\
\hline Buckling & $17(24 \%)$ & $7(7 \%)$ \\
\hline Encircling & $27(38 \%)$ & $3(3 \%)$ \\
\hline ppV+Encircling & $11(15 \%)$ & $17(17 \%)$ \\
\hline with phacoemulsification & $4(6 \%)$ & $10(10 \%)$ \\
\hline with Pars plana lensectomy & $15(21 \%)$ & $27(27 \%)$ \\
\hline
\end{tabular}

Values are presented as n (\%).

* p-value by chi-square test

As the initial surgery, "encircling" accounted for $62 \%$ in the atopy group, and a note of being significantly more frequent than the control group (10\%). In the control group, the most used operation method was ppV alone.

In this study, there were fifteen eyes of atopic patients (21\%) who had cataract surgery at the same time with RD surgery, phacoemulsification in 11 eyes and pars plana lensectomy (ppL) in 4 eyes. In the control group, 27 eyes had cataract surgery together; with phacoemulsification in 17 eyes and ppL in 10 eyes.

The preoperative log MAR VA was 1.14 in the atopy group and 0.96 in control group, therefore there was no statistically significant difference in preoperative VA between two groups. However, the postoperative log MAR VA was 0.80 in the atopic group and 0.34 in control group; postoperative VA was markedly poor in the atopic group ( $P$ value 0.05). There were no statistically significant differences in the preoperative macular off ratio between the two groups. In this sense, the PVR rate was 35\% in the atopy group and $12 \%$ in control group, and reoperation rate was 35\% in the atopy group and $10 \%$ in control group.

Therefore, PVR and reoperation rate were shown to be significantly higher in the atopy group ( $P$ value $0.000,0.001$ respectively),(Table 4$)$. 
Table 4. Pre-operative visual acuity and macula, PVR status \& Post-operative visual \& surgical

prognosis of each group.

\begin{tabular}{lccc}
\hline & Atopy & Non-atopy & P value \\
& & & 0.96 \\
\hline Pre op VA (Log MAR) & 1.14 & 0.34 & 0.294 \\
Post op VA (Log MAR) & 0.80 & $44: 56$ & $0.005^{*}$ \\
Macula ON: OFF (\%) & $34: 37(48: 52)$ & $12 \%$ & 0.615 \\
Rate of PVR (\%) & $35 \%$ & $10 \%$ & $\mathbf{0 . 0 0 0 ^ { * }}$ \\
Re-operation rate (\%) & $30 \%$ & & $\mathbf{0 . 0 0 \mathbf { 1 } ^ { * }}$ \\
\hline -p-value by Mann-Whitney U test and chi-square test &
\end{tabular}

"p-value by Mann-Whitney U test and chi-square test

In this context, the data of RD involving quadrant is present in Table 5. Here it was noted that RD was limited to one quadrant in 6 eyes (8.5\%) in the atopy group, 9 eyes (9\%) in control group. $56.3 \%$ in atopy group and $66 \%$ in control group occupied 2 quadrants. Next, it was revealed that there were $25.4 \%$ in the atopy group and $18 \%$ in control group occupied 3 quadrants. Finally, there was $9.9 \%$ in the atopy group and $7 \%$ in control group, which was shown to invade all four quadrants. In other words, it was noted that the differences between the two groups was not statistically significant (P value 0.213).

Table 5. Number of involved quadrants of RD.

\begin{tabular}{|c|c|c|c|}
\hline & Non-atopy & & P value \\
\hline Involved quadrant & & & 0.213 \\
\hline 1 & $6(8.5 \%)$ & 9 & \\
\hline 2 & $40(56.3 \%)$ & 66 & \\
\hline 3 & $18(25.4 \%)$ & 18 & \\
\hline 4 & $7(9.9 \%)$ & 7 & \\
\hline
\end{tabular}

Additionally, the location of retinal breaks was analyzed at that time (Table 6-a). In the atopy group, retinal breaks were found in the superotemporal and inferotemporal areas as $34 \%$ and $30 \%$, respectively. And in the control group, it was found to have breaks mainly in the superotemporal area (50\%). The type 
of retinal breaks was also analyzed (Table 6-b). The incidence of a horseshoe tear and retinal tear were observed in $30 \%$ and $28 \%$ of the atopic group and $35 \%$ and $27 \%$ of the control group, respectively. However, retinal dialysis was more frequent in the atopy group (16\% in atopy group and $2 \%$ in control group), whereas the lattice hole was more observed in the control group ( $8 \%$ in atopy group, $21 \%$ in control group), (P value 0.000).

Table 6 -a. Location of breaks by fundus quadrant.

\begin{tabular}{lllllll}
\hline & Superotemporal & inferotemporal & superonasal & inferonasal & Unknown & P value \\
\hline Atopy & $24(34 \%)$ & $21(30 \%)$ & $6(8 \%)$ & $10(14 \%)$ & $10(14)$ & \\
Non-Atopy & $50(50 \%)$ & $21(21 \%)$ & $21(21 \%)$ & $6(6 \%)$ & $2(2 \%)$ & \\
& & & & & & $0.001^{*}$ \\
\hline
\end{tabular}

Values are presented as $\mathrm{n}(\%)$.

-p-value by chi-square test

Table 6-b. Types of retinal break.

\begin{tabular}{lcllllll}
\hline & $\begin{array}{l}\text { Retinal } \\
\text { dialysis }\end{array}$ & $\begin{array}{l}\text { Horseshoe } \\
\text { tear }\end{array}$ & $\begin{array}{l}\text { Retinal } \\
\text { tear }\end{array}$ & Lattice hole & $\begin{array}{l}\text { Hole without } \\
\text { lattice }\end{array}$ & Unknown & P value \\
\hline Atopy & $11(16 \%)$ & $21(30 \%)$ & $20(28 \%)$ & $6(8 \%)$ & $6(8 \%)$ & $7(10 \%)$ & \\
Non-Atopy & $2(2 \%)$ & $35(35 \%)$ & $27(27 \%)$ & $21(21 \%)$ & $14(14 \%)$ & $1(1 \%)$ & \\
& & & & & & & \\
\end{tabular}

Values are presented as $n(\%)$.

-p-value by chi-square test

In this study, twenty-eight eyes (39\%) out of 71 eyes in an atopy group, and 26 eyes (26\%) out of 100 eyes in a control group had undergone cataract surgery before the onset of detachment. In the atopy group, RD occurred $1.93 \pm 0.48$ years after cataract surgery. On the other hand, in the control group, RD occurred $4.86 \pm 0.90$ years after cataract surgery. Generally speaking, there was a significant difference of mean period after cataract surgery between the atopy group and control group ( $P$ value 0.002 , Table 7 ). Notably, RD occurred within 6 months after cataract surgery in 9 eyes (32\%) of 28 eyes in atopy group and 4 eyes $(15 \%)$ of 26 eyes in control group ( $P$ value 0.154$)$. RD within 1 year after cataract surgery was significantly common in atopy group; 17 eyes (60\%) of 28 eyes in atopy group and 7 eyes (26\%) of 26 eyes in control group ( $P$ value 0.013$)$. 
Table 7. Mean period after cataract surgery in patients who underwent cataract surgery before.

\begin{tabular}{lccc}
\hline & Atopy & Non-atopy & P value \\
\hline Mean period & $1.93 \pm 0.48$ & $4.86 \pm-0.90$ & $\mathbf{0 . 0 0 2}^{*}$ \\
after cataract surgery (years) & & & \\
Within 1 year; $\mathrm{n}(\%)$ & $17(60 \%)$ & $7(26 \%)$ & $\mathbf{0 . 0 1 \mathbf { 3 } ^ { * }}$ \\
Within 6 month; $\mathrm{n}(\%)$ & $9(32 \%)$ & $4(15 \%)$ & 0.154 \\
\hline
\end{tabular}

Values are presented as mean \pm SD unless otherwise indicated.

•p-value by Mann-Whitney U test

\section{Discussion}

We report in this study the characteristics of RD as associated with atopic dermatitis. Although there are some studies already reported the characteristics of atopic RD, this is the first report compared the characteristics of atopic RD with non-atopic RD. $[2,6,7]$ Moreover, we compare the incidence of RD associated with cataract surgery, tear type or location in an atopy group and non-atopy group.

In Korea, the prevalence of atopic dermatitis is $2.2 \%$ of the total population, $6.9 \%$ of those under 18 years of age, $0.9 \%$ of those over 18 years of age. In this study, $2.7 \%$ of all RD patients had atopy, and there were 1.7 times more male patients than female patients in both groups. Although the prevalence of atopy itself is known to be no difference in relation to gender, male patients were more common than female patients of RD in this study. $[13,14]$ This result was similar to the study from Japan, which showed the incidence of atopy was not different according to sex of the patient but the rate of RD was nearly double in males. [15] Generally speaking, it was noted also in the control group, that male patients were more than female and it is also consistent with previous study which shows men were at a high risk for RD, whether exhibiting atopy or not.[13]

The incidence rate by age was the highest in the 50-60 age group in the control group and second highest in the 20s group, likewise previous study mentioned regarding the bimodal distribution of RD.[16] However, as noted in the atopy group, most of patients were teenagers or twenties. That is, the mean age of onset was 52 in the control group and 23 in atopy group, showing the age of onset in the atopy group was significantly younger ( $P$ value 0.000 ).

In previous studies, it was noted that the bilateral RD accounts for $5-10 \%$ of the total RD.[13] And in this study, $21 \%$ of the atopy group and $1 \%$ of the control group showed an indication of a bilateral 
involvement of RD. Thus, the rate of bilaterality was significantly higher in the atopy group than control group.

There was no significant difference of the mean preoperative refractive index between two groups, but the proportion of high myopia patients with -8 diopters or more was $7 \%$ in the atopic group and $19 \%$ in the control group. The proportion of patients with high myopia was higher in the control group.

There was no difference of RD involving the quadrant or preoperative VA between the two groups. However, postoperative VA was shown to be significantly poorer in the atopy group (log MAR VA 0.80) than in the control group (log MAR VA 0.34), (P value 0.005 ). It shows that VA before surgery was similar, but the prognosis after surgery was significantly worse in the atopy group. It is thought to be due to inflammation caused by atopy itself, delayed wound healing, or increased PVR.

In the control group, the location of the retinal tear was most common in the superotemporal area. And followed by inferotemporal and superonasal area, it was shown to have occupied a similar rate. In the atopy group, the ratio of unknown was $14 \%$ high, as compared to $2 \%$ in control. Most of the tears noted in the atopy group were found in the temporal area, and showed a similar ratio in the superotemporal, inferotemporal area. It suggests that RD may occur due to frequent rubbing of the lateral side of eyes in atopy group. However, the temporal area was also common in the control group, and for this reason further study might be needed.

Chiefly noted, there was no difference in incidence of the right and left eyes. This result shows whether right-handed or left-handed might not be related to the site it where it occurs.

Interestingly, the type of tear was characteristically different in two groups. As was noted in the atopy group, retinal dialysis accounted for $16 \%$, which was thus much higher than $2 \%$ in control group. Previous research has shown that the slapping of the eye acts as a trauma, resulting in retinal dialysis.[17]

Generally speaking, retinal dialysis is the disinsertion of the retina from the vitreous base, and is an uncommon cause of RD, accounting for $8 \%$ to $17 \%$ of the cases.[18, 19] Furthermore, a previous study suggested that retinal dialysis might be associated in many cases with trauma.(20,21), and it shows that trauma caused by eyelid rubbing could be associated with atopic RD. Through this process, the horseshoe tear and retinal tear occupied a similar ratio in both groups, and lattice hole was more frequently found in the control group.

In this respect, PVR is a disease process that follows the proliferation of ectopic cell sheets in the vitreous and/or periretinal area, causing periretinal membrane formation and traction, in patients with RRD.[20] Previous studies show that this disease process occurs in 5\%-10\% of all RRD and is implicated in redetachment after surgery in $75 \%$ of cases, remaining a major barrier to successful repair of RD.[21]. In our study, the PVR rate was $35 \%$ in atopy group and $12 \%$ in control group, indicating a statistically significant difference ( $P$ value 0.000 ). As mentioned above, $R D$ with atopy generally thought to have association with eye rubbing, which often leads to poor peripheral retinal, and it may lead to more incidences of PVR. 
Upon review, the previous study showed about $13 \%$ of patients needed reoperation after the single surgery of RD.[22],[23] In this study, the reoperation rate was 30\% in the atopy group and it was 3 times more than that of the control group (10\%), showing a statistically significant difference (P value 0.001$)$.

Despite the similarity of macular off rate and preoperative VA between two groups, $38 \%$ of atopy group had vitrectomy and encircling together as an initial operative method, which was identified as much higher as compared to $3 \%$ in the control group. In this relation, the surgical method of vitrectomy and encircling together is usually performed in patients with severe PVR or retinal reattachment due to retinal dialysis. However, despite these aggressive treatments, both recurrence rate and postoperative visual prognosis were poor in the atopy group.[24]

At the time of RD occurs, the pseudophakic cases were $40 \%$ in the atopic group and $26 \%$ in the control group. It is emphasized that the mean time between the cataract surgery and onset of RD was $1.93 \pm 0.48$ years in atopy group and $4.86 \pm-0.90$ years in control group. Furthermore, it showed a statistically significant difference between the two groups. Especially, noting the RD within 1 year after cataract surgery was shown to be statistically significant, with an emphasis that $60 \%$ in the atopic group and $26 \%$ in the control group were revealed. This suggests that the risk of RD may increase after cataract surgery in atopy patients, especially up to a year post surgery.

Presumably it is noted that in phakic eyes, the movement of the vitreous body might be limited due to the lens, and in that case the progress of RD could be suppressed to a certain extent. But after the cataract surgery, these mechanisms might be broken and be more susceptible to becoming RD due to the trauma of eyelid rubbing. Also, atopic patients could be more vulnerable to RD due to their specific immune reaction, and the resulting degeneration of the vitreous body.

This study has some limitations. First, there might be a selection bias because we designed our study retrospectively. Second, the sample size in this case was small and follow-up period was relatively short, therefore we plan to analyze additional cases over time.

In conclusion, it is noted that atopic patients have RD in younger age and their prognosis is poor due to the high incidence of PVR or recurrence. Therefore, extensive treatment and management are needed in these instances. Moreover, we found that atopic patients have a much more increased risk of RD after cataract surgery. Thus, careful examination should be performed regularly after the cataract surgery especially in atopic patients for the best patient outcomes.

\section{Declarations}

\section{Availability of data and materials}

The datasets during and/or analyzed during the current study are available from the corresponding author on reasonable request.

\section{Authors' contribution}

Page 12/15 
Youlim Lee and Young-Hoon Park were primarily responsible for experimental concept and design. Youlim Lee and Young-Hoon Park performed data acquisition and analysis, as well as drafting of the manuscript. Woo-kyung Park, Rae-Young Kim, Mirinae Kim, and Young-Gun Park were also involved in data analysis. All authors reviewed and approved the final manuscript.

\section{Competing interests}

The authors declare that they have no competing interests.

\section{Consent for publication}

Not applicable.

\section{Ethical approval and consent to participate}

This study was performed according to the tenets of the Declaration of Helsinki, and the study protocol was approved by the institutional review/ethics boards of the Catholic University of Korea and our hospital. Informed consent was waived owing to the retrospective nature of this study, and patient records were anonymized prior to all records analysis. All protocols were approved by the Institutional Review and Ethics Boards of Seoul St. Mary's Hospital, South Korea.

\section{Funding}

Not applicable.

\section{References}

1. Nutten S. Atopic dermatitis: global epidemiology and risk factors. Ann Nutr Metab. 2015;66 Suppl 1:8-16. doi:10.1159/000370220.

2. Govind K, Whang K, Khanna R, Scott AW, Kwatra SG. Atopic dermatitis is associated with increased prevalence of multiple ocular comorbidities. The Journal of Allergy and Clinical Immunology: In Practice. 2019;7:298-9.

3. Uehara M, Amemiya T, Arai M. Atopic cataracts in a Japanese population. Dermatology. 1985;170:180-4.

4. Nagaki Y, Hayasaka S, Kadoi C. Cataract progression in patients with atopic dermatitis. Journal of Cataract \& Refractive Surgery. 1999;25:96-9.

5. Katsushima H, Miyazaki I, Sekine N, Nishio C, Matsuda M. Incidence of cataract and retinal detachment associated with atopic dermatitis. Nippon Ganka Gakkai Zasshi. 1994;98:495-500.

6. Sasoh M, Mizutani H, Matsubara H, Furuta M, Matsui Y, Yamanaka K-i et al. Incidence of retinal detachment associated with atopic dermatitis in Japan: review of cases from 1992 to 2011. Clinical ophthalmology (Auckland, NZ). 2015;9:1129. 
7. Azuma N, Hida T, Katsura H, Takeuchi S, Danjo S, Tano Y. Retrospective survey of surgical outcomes in rhegmatogenous retinal detachments associated with atopic dermatitis. Archives of Ophthalmology. 1996;114:281-5.

8. Balyeat RM. Complete retinal detachment (both eyes) : With special reference to allergy as a possible primary etiologic factor. Am J Ophthalmol. 1937;20:580-2.

9. COLES RS, LAVAL J. RETINAL DETACHMENTS OCCURRING IN CATARACT ASSOCIATED WITH NEURODERMATITIS. JAMA Ophthalmology. 1952;48:30-9.

doi:10.1001/archopht.1952.00920010033004.

10. Taniguchi H, Ohki O, Yokozeki H, Katayama I, Tanaka A, Kiyosawa M et al. Cataract and Retinal Detachment in Patients with Severe Atopic Dermatitis Who Were Withdrawn from the Use of Topical Corticosteroid. The Journal of Dermatology. 1999;26:658-65. doi:10.1111/j.13468138.1999.tb02068.x.

11. Tan L, Teo L, Lee SY. Case Series: Bilateral Rhegmatogenous Retinal Detachments in Atopic Dermatitis. Optom Vis Sci. 2018;95:621-4. doi:10.1097/OPX.0000000000001244.

12. Machemer R, Aaberg TM, Freeman HM, Irvine AR, Lean JS, Michels RM. An updated classification of retinal detachment with proliferative vitreoretinopathy. Am J Ophthalmol. 1991;112:159-65.

13. Mitry D, Charteris DG, Fleck BW, Campbell H, Singh J. The epidemiology of rhegmatogenous retinal detachment: geographical variation and clinical associations. Br J Ophthalmol. 2010;94:678-84. doi:10.1136/bjo.2009.157727.

14. Wilkes SR, Beard CM, Kurland LT, Robertson DM, O'Fallon WM. The incidence of retinal detachment in Rochester, Minnesota, 1970-1978. Am J Ophthalmol. 1982;94:670-3.

15. Yu JS, Lee CJ, Lee HS, Kim J, Han Y, Ahn K et al. Prevalence of atopic dermatitis in Korea: analysis by using national statistics. J Korean Med Sci. 2012;27:681-5. doi:10.3346/jkms.2012.27.6.681.

16. Kim GJ, Shin MC, Hwang HS, Han SY, Cho B-J. Clinical Features and Surgical Outcomes of Primary Rhegmatogenous Retinal Detachment according to Age. J Korean Ophthalmol Soc. 2017;58:56-61.

17. Takahashi M, Suzuma K, Inaba I, Ogura Y, Yoneda K, Okamoto H. Retinal detachment associated with atopic dermatitis. Br J Ophthalmol. 1996;80:54-7. doi:10.1136/bjo.80.1.54.

18. Kinyoun JL, Knobloch WH. Idiopathic retinal dialysis. Retina. 1984;4:9-14.

19. Chang JS, Marra K, Flynn HW, Jr., Berrocal AM, Arroyo JG. Scleral Buckling in the Treatment of Retinal Detachment Due to Retinal Dialysis. Ophthalmic Surg Lasers Imaging Retina. 2016;47:33640. doi:10.3928/23258160-20160324-06.

20. Sadaka A, Giuliari GP. Proliferative vitreoretinopathy: current and emerging treatments. Clin Ophthalmol. 2012;6:1325-33. doi:10.2147/OPTH.S27896.

21. Pastor JC. Proliferative vitreoretinopathy: an overview. Surv Ophthalmol. 1998;43:3-18.

22. Pastor JC, Fernandez I, Rodriguez de la Rua E, Coco R, Sanabria-Ruiz Colmenares MR, SanchezChicharro $D$ et al. Surgical outcomes for primary rhegmatogenous retinal detachments in phakic and 
pseudophakic patients: the Retina 1 Project--report 2. Br J Ophthalmol. 2008;92:378-82.

doi:10.1136/bjo.2007.129437.

23. Conrath J. [Risk factors for recurrent retinal detachment]. J Fr Ophtalmol. 2007;30:847-51.

24. Heimann H B-SK, Bornfeld N, et al,. Scleral Buckling versus Primary Vitrectomy in Rhegmatogenous Retinal Detachment Study Group. Scleral buckling versus primary vitrectomy in rhegmatogenous retinal detachment: a prospective randomized multicenter clinical study. Ophthalmology. 2007;114:2142-54.

\section{Figures}

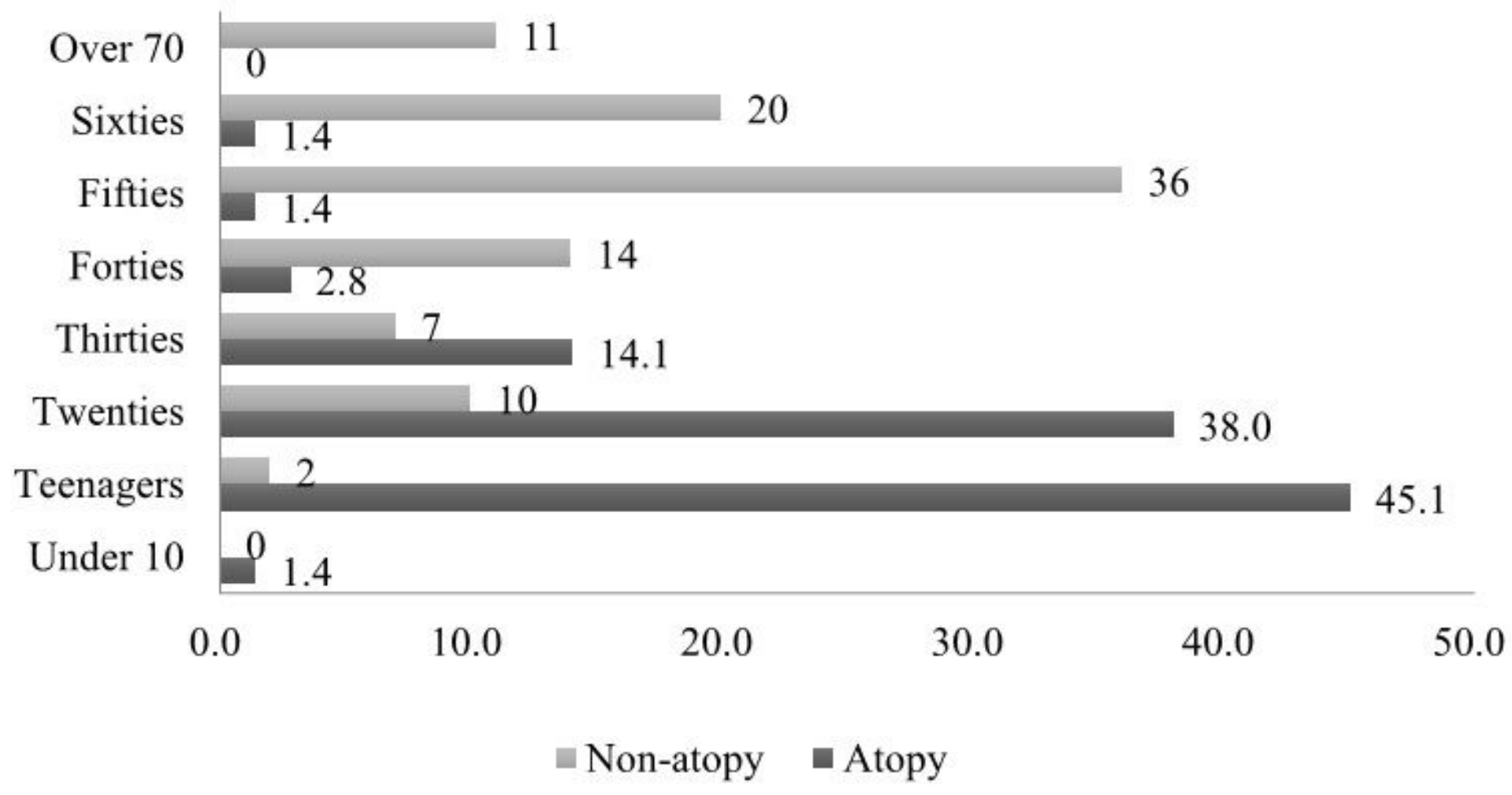

Figure 1

Age of patients. (\%) 